\title{
Medications in Mexico: The Growth and Distribution of Pharmacies in Ciudad Juárez, Chihuahua, Mexico from 1996-2011
}

\author{
Patricia J. Boda \\ Department of Geosciences, Middle Tennessee State University, Murfreesboro, Tennessee, USA \\ Email: pat.boda@mtsu.edu
}

Received June 5, 2013; revised July 9, 2013; accepted August 3, 2013

Copyright (C) 2013 Patricia J. Boda. This is an open access article distributed under the Creative Commons Attribution License, which permits unrestricted use, distribution, and reproduction in any medium, provided the original work is properly cited.

\begin{abstract}
US pharmacies tend to be located at intersections of major cross streets throughout a city, while in the case of Mexican border cities pharmacies are clustered close to US-Mexico border crossings. Presumably this is due to the volume of US clients who frequent the pharmacies. Although the precise number of border crossings to purchase medications is unclear, it is thought to be significant. In the past, patient-based surveys were the primary source for information regarding US residents crossing the border into Mexico for prescription medications. The current study examines the distribution of pharmacies throughout Ciudad Juárez, Chihuahua, Mexico, recording the change in the number of pharmacies over a 15-year period and suggesting the scale of US residents who use Mexican pharmacies. Field research was conducted in 1996 and 2011. Maps showing the location of pharmacies for these two years indicate a clustering of pharmacies within one and one-half miles of the city's two principal border-crossings between the United States and Mexico. Provider-based surveys revealed that the majority of patients who use pharmacies closest to the border were US residents.
\end{abstract}

Keywords: Medication; Cross-Border Health Care; Medical Geography; US-Mexico Border

\section{Introduction}

The border region between the United States and Mexico is an area of shared history but varied cultures, diverse income levels, and dissimilar political environments. Mexican border cities share many similarities to their counterparts across the frontier in the United States (e.g., history, layout, and commercial stores). Typically in the US, drugstores are located in commercial zones found throughout the city, generally on corners of busy thoroughfares, thus providing customers optimal access to their services. Additionally, as metropolitan areas expand, one observes subsequent growth of commercial operations that coincide with the growth of the urban area. As a result, it is expected that the spatial distribution of drugstores in Ciudad Juárez should have a similar pattern to that found in US cities.

According to the Instituto Nacional de Estadistica y Geografica Informacion (INEGI), the Mexican equivalent of the US Census Bureau, Ciudad Juárez had a population of 995,770 in 1995 . By 2010, its population grew by $32.6 \%$ to $1,321,004$ [1]. The actual population of Juárez is difficult to determine because: 1 ) migrants flock into the city seeking employment in the maquiladoras (manufacturing operations in the free trade zone) and are often not counted in the census and 2) many residents leave the city due to violence [2]. Taking these issues into consideration, the population of Juárez in 2011 is thought to be approximately $1,298,004$. Using this number, the \% change from 1996 to 2011 is about $30.4 \%$. To keep pace with the increasing population, one would expect that Juárez's pharmacies to increase in number at about the same rate as the population's increase during the study period.

This study investigates the change in the number of drugstores and spatial distribution of pharmacies in Ciudad Juárez, Chihuahua, Mexico, from 1996 to 2011, with two hypothesis in mind: 1) the number of drugstores is expected to increase at a rate similar to the population growth rate of the city itself and 2) the spatial distribution of pharmacies and/or drugstores is similar to the general pattern found in US cities. 


\section{Background}

In El Paso, as elsewhere, Americans routinely cross the US-Mexico border to obtain a variety of goods and services, including medications. There are three ports of entry into Ciudad Juárez from El Paso: two in the north which directly connect downtown El Paso and Juárez, and one that enters Juárez from El Paso County from the east. "In 2011, more than 3.6 million passenger vehicles, 4.2 million pedestrians and 300,000 commercial vehicles crossed into Ciudad Juárez through the three bridges [3]." Although there are three ports of entry, the two principal border crossings between downtown El Paso and Ciudad Juárez account for the vast majority of crossings. The US Customs and Border Protection Agency recognized these crossing points as the two busiest border crossings in the world in 2011 [4]. Since these two crossing accounted for most of the traffic during the study period, they served as the focus of this research.

Use of particular health care providers (including pharmacies) has been studied for many years and has resulted in a myriad of research. Health care research relevant to this study include the role that poverty and cultural preferences play in a person's decision to use a particular facility $[5,6]$. Other studies have determined that distance and travel time are key factors to utilization [7]. Most researchers agree that in order to use a facility, there must be access to it.

Although access is difficult to define and evaluate, Penchansky and Thomas identified five dimensions of access: availability, accessibility, accommodation, affordability and acceptability. Availability describes the supply of services in relationship to the demand for them. Accessibility defines the geographic barriers to receiving such services such as distance, transportation, and travel time to the facility. Accommodation refers to the degree that services meet the needs of patients and include hours of service, waiting times, appointments and scheduling. Affordability denotes the cost of services and acceptability articulates how the provider interacts with the patient on a personal level. Acceptability includes potential barriers such as gender, ethnicity, language and sexual orientation [8]. Each dimension can interfere with true access by creating barriers that limit utilization of services.

Previous research regarding US patients who cross into Mexico for prescription medications focused on the volume and cultural characteristics, [9-13] while others have examined the dangers and legal issues associated with doing so [14-18]. Specifically, patient-based surveys were used to describe and identify why US residents cross into Mexico from Arizona, California, New Mexico, and Texas to buy prescription drugs and medications. In addition, Homedes and Ugalde found that those who crossed to Mexico for health care faced a variety of challenges including administrative, legal and cultural barriers [19].

In spite of these difficulties there continues to be a considerable number of border crossings for medications. Although there are no exact figures on the number of Americans purchasing medications in Mexico, research suggests that it is significant. A Texas Tech Medical University study showed that $80 \%$ of its El Paso patients purchased prescription-type medication in Mexico without a prescription [9] Escobedo and Cardenas in their 2006 study conducted in New Mexico found that 22\% of that State's residents purchased medications in Mexico [13]. In Laredo, Texas, approximately two-in-five (41\%) Hispanics living in Laredo traveled to Mexico to buy prescription drugs and/or medications [12], while in another borderland study, $28 \%$ of California residents interviewed for the report purchased medications in Mexico [11]. In October and November of 2007, researchers who conducted over 2500 telephone interviews and found that $32.5 \%$ of respondents from El Paso had crossed into Juárez for some type of health care during the previous two years [20].

Several factors compel US residents to cross into Mexico to purchase prescription drugs/medications. The hybrid nature of borderland populations is a factor [21]. Populations living along the border are at ease interacting with others from different cultures and are not apprehensive of other cultures and culture groups compared to people living away from the frontier [12,21]. The primary reason for crossing the border for medications however is cost savings. In 2001 Macias and Morales conducted a survey at a health fair in southern Los Angeles County, approximately 140 miles from the US-Mexico border. They found that while only 14\% of the respondents had crossed the border for medical care in the previous year, $80 \%$ of those who did were uninsured. $28 \%$ of the respondents crossed the border did so to purchase medications, and $90 \%$ of that group stated they had no insurance. The low cost of medical services and medications was the most common reason given for seeking services in Mexico by $70 \%$ of the respondents [11].

\section{Data Sources and Research Methods}

The current study is an extension of work conducted previously in El Paso, Texas and Ciudad Juárez, Chihuahua, in 1996 and in 2007 [22,23] that documented concentrations of certain healthcare-related facilities near the border crossing points in northern part of Ciudad Juárez. Mapping and provider-based surveys were utilized to examine trends. They indicated that 1 ) the number of pharmacies growing at a rate faster than that of the population growth rate itself-suggesting that people living in the US visited Juárez, creating the demand for more pharmacies and 2) the new drugstores built during the study period have joined earlier facilities, clustering 
within one and one-half miles of the two main border crossings to better serve their primary customers, US residents.

The primary source for identifying pharmacies was the Yellow Pages in El Paso and Ciudad Juárez, 1996 and 2011. In both years, pharmacies were defined as facilities that were advertised under the heading "Pharmacy."

Street files for Ciudad Juárez were purchased through Instituto Nacional de Estadistica y Geografica Informacion (INEGI). The file contained street and colonia (similar to census tracts) data however, street segments did not have any coordinate reference system included, and in addition it did not contain address ranges, which prevented geocoding operations. Although address matching was not possible, the street and colonia files were imported into ArcMap for mapping purposes only. Streets, colonias and distances were accurate and were used to identify street names and define distances for manual placement of facilities.

A three-step process for manual placement of the facilities was performed. First, the street name of each facility was identified and located in the street file. A second query was performed to locate that section of street within a polygon, in this case, a colonia since this information was provided by the Yellow Page advertisement. The third step in the location process placed pharmacies in a particular order on the street and within the colonia as follows

*If a facility were located on a short street, that is 1 - 5 blocks in length, then the site was located in the middle of the street section.

*If more than one facility were located on any length street, then locations were divided evenly along that street.

As a result, pharmacies were located on the correct street and in the correct colonia, however the exact location of each facility on the street itself could be slightly inaccurate because each was manually placed in ArcMap. Since exact locations of many facilities were known, accuracy of the procedure of interpolation was verified by overlaying "interpolated" locations with known locations. While some facilities resulted in less than a $100 \%$ match, most were within one block of exact locations and only one was located more than two blocks from its actual site. This process proved to be a useful method for locating pharmacies in those cases where address matching was unavailable.

Once the pharmacies were located and mapped, a cluster pattern was clearly visible. After identifying this cluster pattern, Ciudad Juárez for the purpose of this study was divided into three zones based on distance from the two principal border crossing points: one and one-half miles (referred to as the border zone), one and one-half miles to three miles (referred to as the mid- zone), and greater than three miles (referred to as the outer zone).

After establishing the three zones, interviews were conducted with a sampling of pharmacy owners/workers in each zone. At least ten \% of the pharmacies in each of the three zones were selected for on-site interviews. The same questionnaire was used in 1996 and 2011 and whenever possible, sites interviewed in 1996 were interviewed in 2011. Interviews were conducted in a conversational style, employing Yin's methodology [24], which was designed to work more effectively when interviewing people from a different culture.

\section{Findings}

\subsection{Number of Pharmacies}

The total number of pharmacies in Juárez grew from 238 in 1996 to 348 in 2011, an increase of 110 new pharmacies or $46.2 \%$ as is seen in Table 1. The border zone (one and one-half miles from the principal border crossings), itself, saw an increase of 90 new pharmacies, accounting for $81.8 \%$ of all new pharmacies in Juárez over the 15year period.

The distance between the two main border crossing points is approximately two miles, and with the border zone of one and one-half miles from each crossing point, the border zone has an area of approximately 7.5 square miles. Since the majority of the new facilities opened in Juárez during the study period are in the border zone, it strongly suggests that these facilities serve US citizen demand for pharmacy services.

\subsection{Distribution of Pharmacies}

The dramatic increase in the number of pharmacies in the border zone is further illustrated in Figures $\mathbf{1}$ and $\mathbf{2}$ and again indicates that US customers were the reason for these new facilities being built within one and one-half miles of the two principal border crossings (see Figures 1 and 2). As shown in Figure 1, in 1996 facilities are closely clustered near the US border crossing points in the northern part of the city. The remainder of the city has a much sparser distribution.

Although pharmacies are found in other parts of the city, a clustering and concentration is in the north-near the two US border crossing points. This was the case in 1996 and remains so in 2011. The map in Figure 2 de-

Table 1. Number of pharmacies 1996-2011. Number of interviews conducted shown in parentheses ( ).

\begin{tabular}{ccc}
\hline Pharmacies & $\mathbf{1 9 9 6}$ & $\mathbf{2 0 1 1}$ \\
\hline Total Number & $238(53)$ & $348(62)$ \\
Border Zone & $199(38)$ & $289(43)$ \\
\hline
\end{tabular}




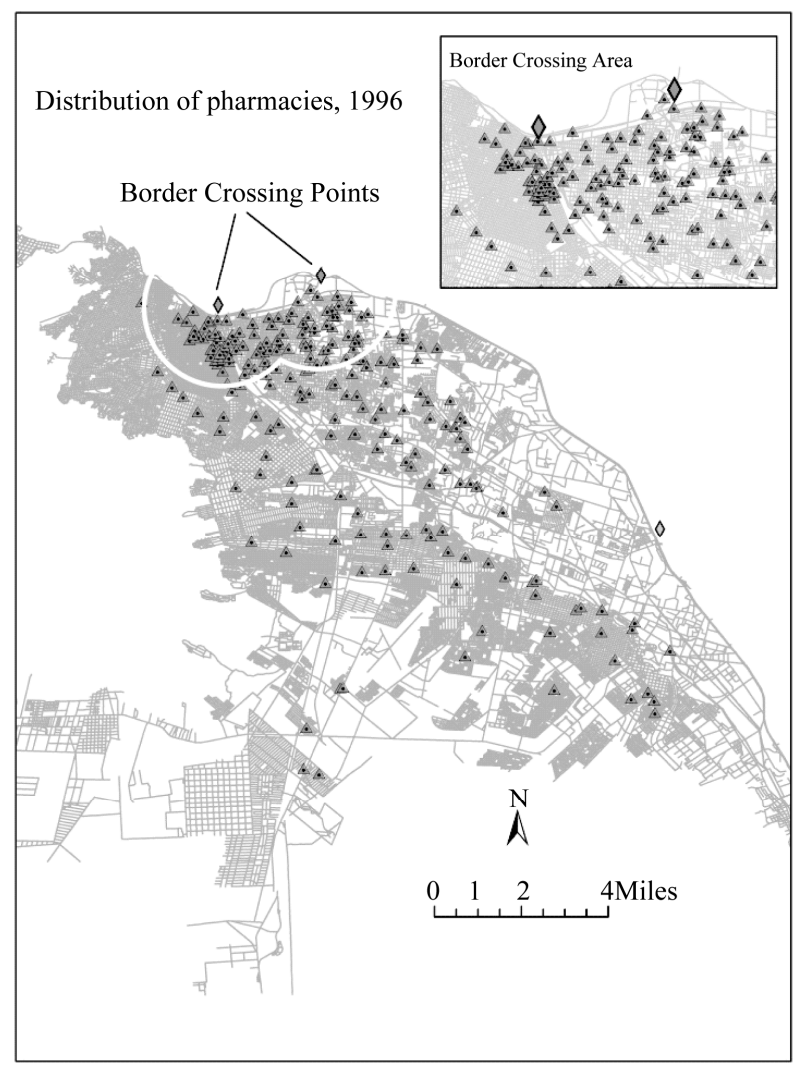

Figure 1. Distribution of pharmacies, 1996.

monstrates visually the preponderance of pharmacies within one and one-half miles of the border crossings, and the US customers. As the inset maps show, in both years the facilities tended to be located near the border crossing points and along the main streets leading from those entry points, and close to their clients. (Figure 2).

\subsection{Use by US Residents}

Assuming that Juárez pharmacies locate their facility near their patients, as do most providers, the distribution of drugstores in Juárez indicates propinquity to US patients. Mexican dentists locate their facilities as a convenience to Americans making the trip from El Paso across the border into Juárez. The demand generated by US residents is significant. In the border zone, pharmacies' customers from the US comprised a much higher \% age than those in either the mid- or outer-zones. As Table 2 illustrates, all those reporting highest \%ages of US clientele were located in the border zone (Table 2).

In 2011, not all pharmacies within one and one-half miles of the border crossings reported $100 \%$ use by US residents, however all pharmacies that reported $100 \%$ of their clients were US residents were within the one and one-half miles of the border crossings. In other words, the further these facilities are from the border, and US base (El Paso), the fewer customers are from the US. The

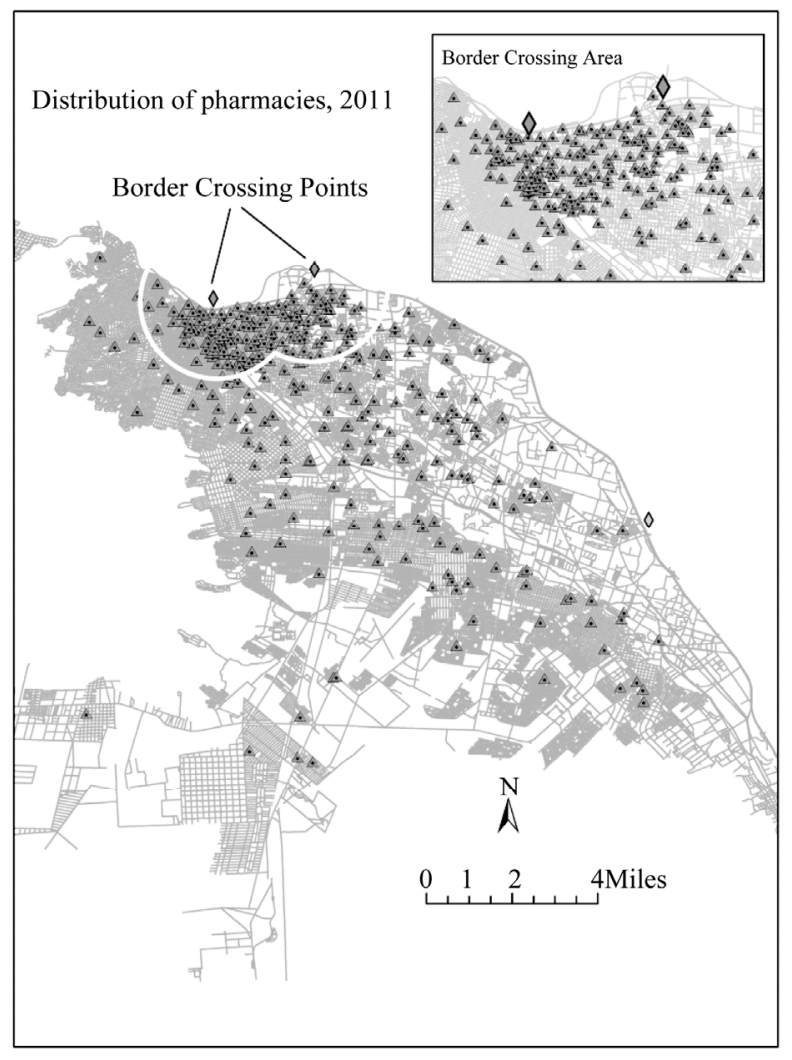

Figure 2. Distribution of pharmacies, 2011.

Table 2. Percentage of pharmacy clients from US by zone.

\begin{tabular}{ccc}
\hline Pharmacies & $\mathbf{1 9 9 6}$ & $\mathbf{2 0 1 1}$ \\
\hline Border zone & $75-90$ & $75-100$ \\
Mid-zone & $20-30$ & $25-40$ \\
Outer zone & $0-5$ & $5-10$ \\
\hline
\end{tabular}

dramatic decline is use is clearly expressed in Figure 3.

\section{Summary and Discussion}

\subsection{Summary}

This study examined two hypotheses. The first one anticipated that drugstores during the study period, 19962011, would increase in number at a rate similar to the population growth rate of the city itself, suggesting as the city grew so too would the number of pharmacies. Ciudad Juárez's population increased at an adjusted rate of about $30.4 \%$, while pharmacies increased by $46.2 \%$ over the same period, a difference of $15.8 \%$ age points. This difference suggests that the pharmacies served more than the just the residents of Juárez alone. US patients help to explain the increase in drugstores, especially when the neighborhoods closest to the border crossing points are examined. The number of pharmacies in the border zone increased $68.9 \%$. Furthermore, $81.8 \%$ of new pharmacies in the city were in the border crossing area. The data 


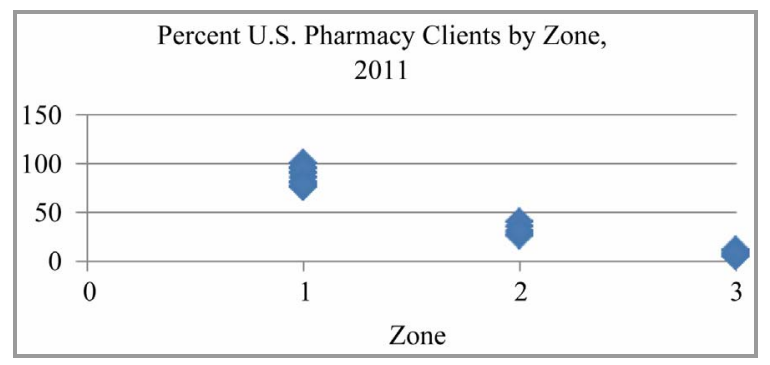

Figure 3. Use of pharmacies by US residents by zone.

from the interviews and the number of pharmacies clustered near the crossing points confirms that the primary customers for these services are US residents.

The second hypothesis stated that the spatial distribution of pharmacies and/or drugstores should be similar to the general pattern found in US cities, drugstores are located fairly uniformly in commercial zones throughout the city. In Ciudad Juárez the spatial distribution of pharmacies is decidedly clustered close to the two principal US-Mexico crossing points, which is very different from the US norm. In fact, $83.5 \%$ of pharmacies in Ciudad Juárez are located within the border area (one and one-half miles of the two border crossing points). So the general distribution of drugstores has not changed, even while the number of facilities has increased considerably. Pharmacies continue to cluster within one and one-half miles of the two principal border-crossing points. As the maps and tables display, more than $83.0 \%$ of drugstores are concentrated within one and one-half miles of the US border. This was true in 1996 and remains the so in 2011, due to the high volume of US residents who cross the border seeking medications.

Interviews with drugstore owners in all three of the zones confirm that Americans frequent pharmacies closest to the two principal border crossings. Since the primary customers near the border are US residents, it is reasonable to conclude that the concentration of these facilities close to the border were built to serve US residents traveling to Juárez to purchase medications. Since there are more facilities near the border and these facilities reported a higher \%age of use by US patrons, it seems to suggest that there were more US residents crossing into Juárez for medications in 2011 than in 1996.

In summary, the city of Ciudad Juárez is still experiencing a great number of US residents who cross into Mexico for their medications. Pharmacists in the city have responded to this continued, increasing demand by opening new facilities near the border to serve this demand. The increase in the number of facilities is due to the US demand based on three pieces of evidence: 1) the pattern of distribution, 2) the growth in the number of border zone facilities that is disproportional to the growth of the city's population and 3) the changes in reported use by US residents.

\subsection{Discussion and Future Research}

During the study period, it is clear that US residents continue to access pharmacies in Ciudad Juárez. Access remains an elusive term however. Applying Penchansky and Thomas's five dimensions of access (availability, accessibility, accommodation, affordability and acceptability) [8] to the case of Ciudad Juárez, the present study revealed that the primary reason for US residents to cross the border and enter a foreign country for medications was affordability, followed closely by the accommodation of Mexican pharmacy owners locating their facilities close to the entry points from El Paso into Juárez. The cost savings are significant. Pharmaceuticals in Juárez are often $50 \%$ to $75 \%$ less than in the US, drawing many US residents to drugstores in the city. Since the savings are significant, barriers, such as proximity to home, become less important. It is clear that many US residents are willing to tolerate the inconvenience of accessibility by spending additional time to travel a greater distance, in order to receive services at a low cost (affordability) that are offered by many providers (availability) who accommodate their schedules by offering extended office hours (accommodation), regardless of language, ethnicity or culture (acceptability).

Future research on this topic should attempt to address several topics. First, participants were not asked if the customers had a prescription in hand from either a Mexican or US physician. Nor were providers asked to describe the ethnicity, gender, or age of their clients. These are important matters to examine in order to identify populations that are most likely to cross into Juárez, and other border communities, for health care services. Are second- and third-generation Mexican-Americans less likely to cross than newcomers as Su and Wang documented, [21]? Are the elderly who are often priced out of US health care, as Families USA Foundation pointed out, more likely to make the trip into Juárez [10]?

Another topic that needs examination is the development of a modified gravity model that incorporates a variety of purchasing factors, including travel time/distance, to determine the areal extent of those who cross into Mexico for health care. This would also provide information on the extent of the economic impact that border crossing has had on the businesses in the US First, what effect does this border crossing for medications have on neighboring pharmacies in US cities, such as El Paso, Las Cruces, Yeleta and other cities along the border? And what is the areal extent for these services? Presumably there would be higher numbers people living in El Paso, who cross the border into Juárez to buy medica- 
tions, but how far into the US does the lure of inexpensive medications extend?

\section{Acknowledgements}

Special thanks and acknowledgement is extended to Dr. James W. Harris, Metropolitan State University of Denver for his insightful review and suggestions prior to submission of this work.

\section{REFERENCES}

[1] Instituo Nacional de Estadistica y Geografica Informacion (INEGI), “Catalogue of Population,” Housing and Household Data, 2010.

[2] R. Carroll, "Mexico Drug War: The New Killing Fields," The Guardian, 3 September 2010.

[3] City of El Paso, 2013. http://home.elpasotexas.gov/bridges/

[4] US Customs and Border Protection Agency, "U.S. Border Patrol Fiscal Year 2011 Profile Report,” Department of Homeland Security, 2011.

[5] C. Brach and L. Fraser, "Can Cultural Competency Reduce Racial and Ethnic Disparities? A Review and Conceptual Model,” Medical Care Research and Review, Vol. 57, Suppl. 1, 2000, pp. 181-217.

[6] M. Welch, "Required Curriculum in Diversity and CrossCultural Medicine: The Time Is Now," Journal of the American Medical Women's Association, Vol. 53, Suppl. 3, 1998, pp. 121-123.

[7] J. Fortney, N. Chambers, M. Cody and C. Beck, "Geographic Access and Service Use in a Community-Based Sample of Cognitively Impaired Elders,” Journal of Applied Gerontology, Vol. 21, No. 3, 2002, pp. 351-367. doi:10.1177/073346480202100305

[8] R. Penchansky and J. W. Thomas, "The Concept of Access: Definition and Relationships to Consumer Satisfaction,” Medical Care, Vol. 19, No. 2, 1981, pp. 127-140. doi:10.1097/00005650-198102000-00001

[9] P. R. Casner and L. G. Guerra, "Purchasing Prescription Medication in Mexico without a Prescription. The Experience at the Border," Western Journal of Medicine, Vol. 156, No. 5, 1992, pp. 512-516.

[10] "Going to Mexico: Priced out of American Health Care," Families USA Foundation, Washington DC, 1992, p. 3.

[11] E. P. Macias and L. S. Morales, "Crossing the Border for Health Care," Journal of Health Care for the Poor and Underserved, Vol. 12, No. 1, 2001, pp. 77-87. doi:10.1353/hpu.2010.0643
[12] M. Landeck and C. Garza, "Utilization of Physician Health Care Services in Mexico by U.S. Hispanic Border Residents,” Health Marketing Quarterly, Vol. 20, No. 1, 2002, pp. 3-16. doi:10.1300/J026v20n01_02

[13] L. G. Escobedo and V. M. Cardenas, "Utilization and Purchase of Medical Care Services in Mexico by Residents of the United States of America, 1998-1999," Pan American Journal of Public Health, Vol. 19, No. 5, 2006, pp. 300-305. doi:10.1590/S1020-49892006000500002

[14] "Purchasing Prescription Drugs from Foreign Countries and Reducing Drug Costs,” California State Board of Pharmacy, Pamphlet, 2003.

[15] “Attitudes about Importing Medication,” National Association of Chain Drug Stores, Pamphlet, NACDS Foundation, Public Opinion Research, Alexandria, 2003.

[16] J. A. Pagan, S. Ross, J. Yau and D. Polsky, "Self-Medication and Health Insurance Coverage in Mexico," Health Policy, Vol. 75, No. 2, 2005, pp. 170-177. doi:10.1016/j.healthpol.2005.03.007

[17] "Consumer Information: Buying Medications from Outside the United States,” US Food and Drug Administration, Pamphlet, Center for Drug Evaluation and Research, Silver Spring, Maryland, 2006.

[18] US Customs and Border Protection Agency, "Buying Prescription Medicine from Internet Foreign Pharmacies," 2010.

[19] N. Homedes and A. Ugalde, "Globalization and Health at the U.S.-Mexico Border," American Journal of Public Health, Vol. 93, No. 12, 2003, pp. 2016-2022. doi:10.2105/AJPH.93.12.2016

[20] T. Byrd and J. Law, "Cross-Border Utilization of Health Care Services by United States Residents Living near the Mexican Border," Revista Panamericana de Salud Publica, Vol. 26, No. 2, 2009, pp. 95-100. doi:10.1590/S1020-49892009000800001

[21] D. Su and D. Wang, "Acculturation and Cross-Border Utilization of Health Services,” Journal of Immigrant and Minority Health, Vol. 4, No. 4, 2011, pp. 563-569.

[22] P. Schwarz, "International Health Care Refugees of the U.S.-Mexico Border,” M.A. Thesis, University of Minnesota, Minneapolis, 1996.

[23] P. Boda, "International Trade of Health Care along the U.S.-Mexico Border: The Case of El Paso and Ciudad Juárez,” Ph.D. dissertation. University of Minnesota, Minneapolis, 2007.

[24] R. Yin, "Interviews as Conversations,” In: R. Burgess, Ed., In the Field, Harper Collins, New York, 1984, pp. 83-99. 